\title{
Screening of Asymptomatic Passengers' Departure from Qatar: A Retrospective Observational Study
}

\author{
Amit Varma ${ }^{1}$, Layla Abdulla AlDahnaim¹, Abdullah Al Naama ${ }^{1}$, Sakthikumar Vedasalam ${ }^{1}$, Abdul \\ Rafi Mohammed ${ }^{1}$, Muneer Abubaker ${ }^{1}$, Shameer Mohammed ${ }^{1}$, Amine Souissi ${ }^{2}$, Sarah Musa $^{1}$, Bassem \\ Mkaouer $^{2}$, Helmi Ben Saad ${ }^{3}$ and Ismail Dergaa ${ }^{1,2 *}$ \\ ${ }^{1}$ Primary Health Care Corporation (PHCC), Doha P.O. Box 26555, Qatar \\ ${ }^{2}$ National Observatory of Sport, Research Unit "Physical Activity, Sport and Health", Tunis, Tunisia" \\ ${ }^{3}$ Farhat HACHED Hospital, Research Laboratory "Heart Failure, LR12SP09”, University of Sousse, Sousse, Tunisia
}

\begin{abstract}
Introduction: International travel has played a significant role in the 2019 coronavirus disease pandemic (COVID-19) and especially the spread of the new strains all over the world. To prevent the spread of the causative agent, many nations and airline companies around the world have applied travel restrictions policies as a ubiquitous tool to limit further spread of COVID-19. A negative reverse-transcriptase polymerase chain reaction (RT-PCR) performed by a clinical laboratory within 48-72 hours of departure has been a common requirement.
\end{abstract} PCR.

Aim: To assess the relative frequency of detection of COVID-19 positive rates amongst asymptomatic passengers through RT-

Methods: In this retrospective study, we analysed data from medical e-records of 2,172 asymptomatic passengers flying out from Qatar to their respective countries in the month of July' 2020. These passengers had a RT-PCR COVID-19 test done 72h prior to their travel date at a designated COVID Centre in Qatar.

Results: From the total of 2,172 asymptomatic passengers (63\% males), 57 (2.62\%) were COVID-19 positive, with 20 (35.09\%) passengers having a high viral load signifying high infectivity measured by the cycle threshold values (CT value $<30)$.

Conclusion: These findings highlight the importance of recognising potential COVID-19 positive patients to help mitigate the global impacts of COVID-19 by preventing travel of infective patients, especially with the emergence of the new COVID-19 strains and hence limiting the spread of this pandemic.

KEYWORDS: Cycle threshold; Herd immunity; Public health; RT-PCR, SARS-Cov-2; Travellers

\section{INTRODUCTION}

The pandemic of coronavirus disease 2019 (COVID-19) has had a significant impact on travel both within and between countries, as overseas travel has been a significant driver of the pandemic [1]. To limit the spread of the causative agent, many countries and local regions have implemented travel bans and restrictions [2]. The emergence of COVID-19 variant strains, some of which are associated with an increased risk of transmission, has added new urgency to efforts to limit the virus's global spread [3]. Till July $6^{\text {th }}$, 2021, COVID-19 infections have been linked to an estimated 185 million cases and four million deaths worldwide [4]. Due to the variability in restrictions between countries that may change over time, international travel presents a particular challenge during the COVID-19 pandemic. Strict travel restrictions were in place
Quick Response Code:



Address for correspondence: Ismail Dergaa, Primary Health Care Corporation (PHCC), Doha P.O. Box 26555, Qatar. ORCID: 0000-0001-8091-1856

Received: September 22, 2021 Revised: October 20, $2021 \quad$ Accepted: October 26, 2021

How to cite this article: AA Varma, LA AlDahnaim, A Al Naama, S Vedasalam, AR Mohammed, M Abubaker, S Mohammed, A Souissi, S Musa, B Mkaouer, H Ben Saad, I Dergaa. Screening of Asymptomatic Passengers' Departure from Qatar: A Retrospective Observational Study. 20213(5) OAJBS.ID.000341. DOI: 10.38125/OAJBS.000341 
with stringent active surveillance since January 2020, after Wuhan was identified as the epicentre of the COVID-19 [5]. Many other countries followed suit after this, imposing travel restrictions and quarantining passengers arriving from China $[1,6]$.

The course of action at the time seemed to have controlled the transmission of COVID-19 outside of China until February 2020, but manifold factors hinted at on-going risk, with evidence emerging about the increasing infectivity of the virus during the asymptomatic incubation period of COVID-19 with reports emerging of passengers eluding rules and regulations in place at the time $[7,8]$. At the beginning of the pandemic, many countries have imposed passenger screening at airports by temperature and symptom-checks in an effort to halt the spread of COVID-19, especially to developing countries that lack health infrastructure and resources to combat it effectively [1].

Developed countries have escalated control measures beyond screening-based containment policies and have placed travel restrictions from high-risk infected countries and quarantined inbound passengers for a duration ranging between one to two weeks [1]. Meanwhile, in many other countries, symptom-screening remains the primary barrier to case importation [9]. Even in countries with the resources to enforce quarantine measures, implementing arrival screening would be the first logical response as the pandemic continues to spread globally. It has been estimated that basic symptom-based screening missed more than half of infected people, thus highlighting the factors leading to screening successes and failures $[8,10]$.

Most cases missed by screening were fundamentally undetectable because they had not yet developed symptoms and were unaware of being exposed to the virus $[2,10]$. It has thus become pertinent to acknowledge that basic screening method is not very systemised and effective in order to curb the transmission of COVID-19 [10,11].

Due to the high infectivity of COVID-19 and the rapid emergence of many new strains, accurate diagnostic methods are urgently required to identify, isolate and treat patients as soon as possible, thereby reducing morbidity and mortality rates and the risk of public transmission and mostly mitigating the emergence of stronger strains [12].

Reverse-transcriptase polymerase chain reaction (RT-PCR) test of oropharyngeal and nasopharyngeal mucus samples has been considered the near-gold standard test in the detection of COVID-19 globally as it was the earliest established laboratory diagnosis method with an average sensitivity of $56.8 \%$ and much higher specificity rate of $99.5 \%[13,14]$. This has hence been recommended and considered as the preferential screening method for passengers too. The Ministry of Public Health (MOPH) in Qatar in collaboration with the embassies of various other countries and airlines have since directed all passengers to have a "fit to travel certificate (FTC)" with a RT-PCR COVID-19 negative result, $72 \mathrm{~h}$ prior to the departure date and time, as a mandatory requirement for boarding any outbound flight from Qatar [1].

The aim of this study was to assess the relative frequency of detection of positive rates of RT-PCR COVID-19 in a cohort of 2,172 asymptomatic passengers departing from Qatar. This was to highlight the importance of RT-PCR COVID-19 screening test to identify infective individuals among asymptomatic passengers, thus helping prevent the spread of this pandemic to other countries.

\section{POPULATION AND METHODS}

\section{Patients and Data Collection}

After review and approval by the institutional review board at Primary Health Care Corporation (Ref No. PHCC/ DCR/2020/08/091), a retrospective observational study was conducted to include all passengers screened for COVID-19 to obtain the FTC as per the MOPH requirement, at Rawdat al Khail Health Centre (RAK-HC) which is a designated government COVID-19 centre in Qatar for the month of July' 20. Only asymptomatic passengers were included in this study. Clinical and demographic data were collected from the medical e-records of the passengers after all identifiable variables being removed as per the research study requirements.

\section{RT-PCR Testing}

Passengers were instructed by the airlines and their respective embassies to get an FTC with a COVID-19RT-PCR negative report, 72h prior to the travel time. Nasopharyngeal and oropharyngeal swab specimens were obtained for RT-PCR testing from all passengers presenting to RAK-HC during the study period. Collection of the samples was performed by trained medical personnel at the health centre.

The samples were transferred in viral transport media immediately after collection to microbiology and molecular pathology laboratory at Hamad Hospital, which is the government designated testing laboratory in Qatar. The results were released in about 6-8 $\mathrm{h}$ after the samples were analysed and the passengers were instructed to come back to RAK-HC the following day after the swab test to collect their results and certificates.

The cycle threshold (CT) value is essentially the number cycles of doubling amplification at which target detection has occurred [15]. It is defined as the number of cycles of amplification (using RTPCR) required for the fluorescence of a PCR product to be detected crossing a threshold, which is above the background signal. According to the CT values, the COVID-19 patients were divided into two groups: i) A group with a high viral load (i.e., CT value $<30$ ), signifying high infectivity and hence a much higher risk of infecting potential fellow passengers if they weren't screened in the first place and detained from travelling [15]; and ii) A group with a low viral load (i.e., CT value $\geq 30$ ); indicating low infectivity [15].

\section{Statistical Analysis}

Data of the baseline characteristics are reported as mean \pm standard deviation (SD) and frequency. The normality of distribution estimated by the Kolmogorov-Smirnov test was not acceptable for all variables. Therefore, Pearson Chi-Square was applied to compare different diagnostics, sex and nationality. The results were considered significantly different when the probability was $\leq 0.05$. Statistical analyses were performed using the software package SPSS version 20.0 [SPSS, Chicago, IL, USA].

\section{RESULTS}

Out of a total of 2,172 passengers (63\% male), i) 41 (1.89\%) were excluded from the study analysis as they had an "inconclusive result" and were instructed to repeat the test as per the RT-PCR screening protocol and ii) $57(2.62 \%)$ were tested positive for COVID-19. Figure 1 presents the numbers of COVID-19 positive passengers ( $n=57,11$ females) amongst different nationalities. Their mean \pm SD (minimum-maximum) age and CT values 
were $34 \pm 13(3-66)$ years and $31 \pm 5(18-38)$. Of the 57 positive passengers, $20(35 \%)$ had a high viral load (mean \pm SD age $=36 \pm 14$

years) and $37(65 \%)$ had a low viral load (mean \pm SD age $=34 \pm 13$ years) (Figure 2).

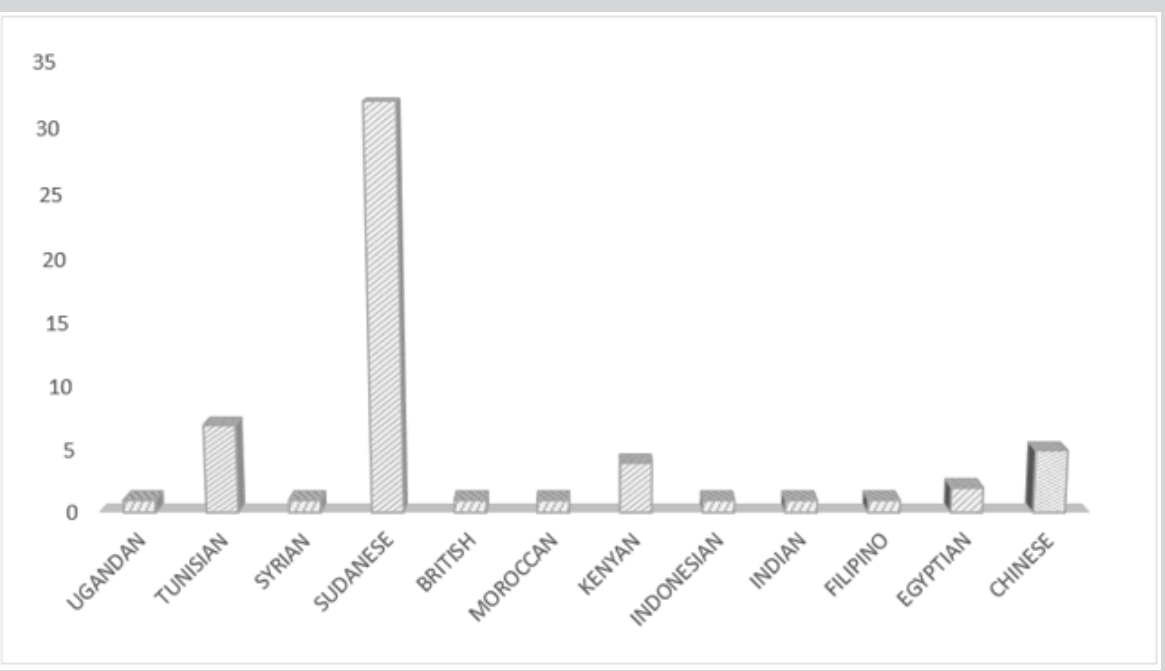

Figure 1: The numbers of COVID-19 positive passengers amongst different nationalities $(n=57)$.

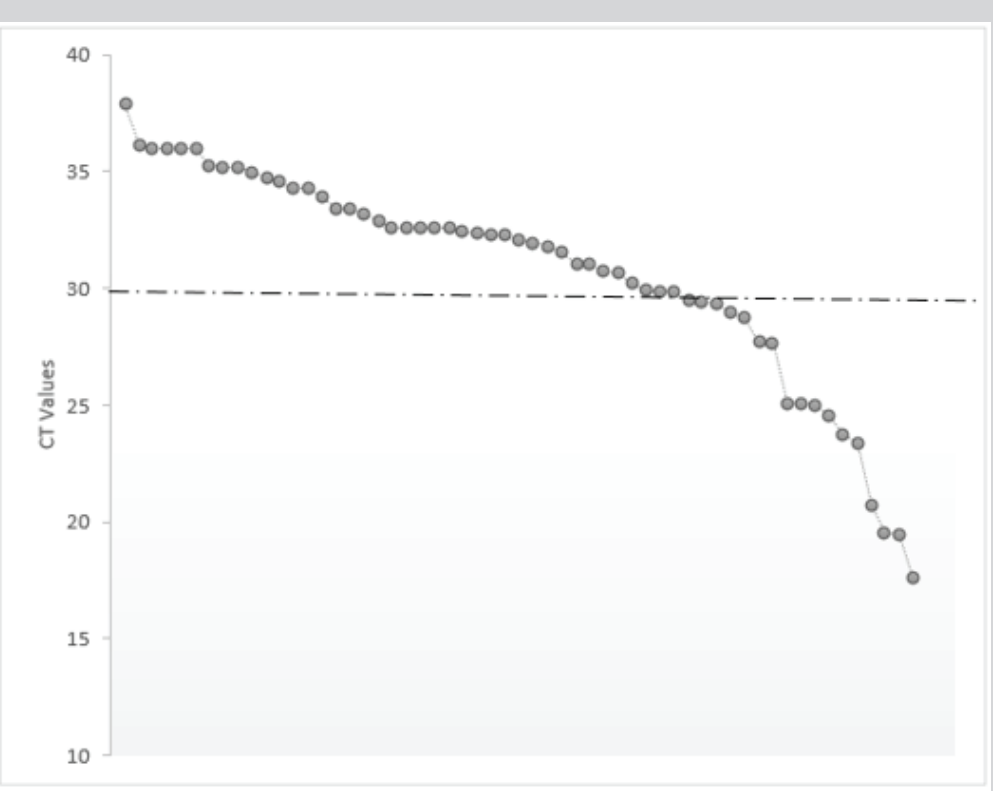

Figure 2: Distribution of cycle threshold value (CT) amongst COVID-19 positive passengers [ $n=57,20$ passengers with a high viral load (i.e., CT value $\leq 30$ ) and 37 passengers with a low viral load (CT value >30)].

\section{DISCUSSION}

The main result of the present study was that out of a total of 2,172 asymptomatic passengers, 57 (2.62\%) were tested positive for COVID-19. Rigorous screening protocol of asymptomatic passengers departing from Doha has helped to prevent the spread of COVID-19 to 12 other countries amongst the cohort tested.

The emergence of COVID-19 posed a global threat and challenge to humanity with the healthcare system across the planet being under considerable stress especially with the emergence with new strains [1]. To curb the spread of this pandemic, countries had to impose strict lock-down measures. However, as countries gradually re-opened to economy and mandatory travel must be allowed for, the previously implemented symptomatic screening with questionnaires and temperature checks at airports being the sole screening procedures in place was tenuous and insufficient. As a result, COVID-19 has now spread to over 210 countries across the world [4].

As a gold standard pre travel screening tool, COVID-19 diagnostic testing primarily employs RT-PCR on a variety of respiratory tract specimen types [14]. For detecting active infection, RT-PCR testing has the highest sensitivity and specificity of any available diagnostic method.

Antigen tests, including some point-of-care and at-home tests, have recently emerged as an option for acute diagnosis $[14,15]$. Antigen tests, on the other hand, have lower sensitivity, particularly for asymptomatic disease and most have lower specificity. Serologic assays for antibodies against COVID-19 are another option, though they are far more useful for defining epidemiology with seroprevalence studies than for clinical diagnosis [16-18]. 
In order to improve international travel safety, destination countries and/or airlines may require COVID-19 testing in addition to other measures $[8,19]$. A COVID-19 RT-PCR performed by a clinical laboratory within 48 to 72 hours of departure, with official documentation of the results, is a common requirement. This requirement can be difficult to meet if test availability is limited and/or results are returned in a timely manner. COVID-19 testing capacity varies greatly by state in the United States, with testing performed by a variety of entities including hospital laboratories, commercial reference laboratories, public health facilities and public-private partnerships. In the United States, supply chain and turnaround time issues with COVID-19 testing have been a recurring issue $[20,21]$. Antigen tests, to the authors' knowledge, have not been an acceptable alternative to RT-PCR testing for international travel, though they may be useful for intra-country travel [22]. Regarding COVID-19 RT-PCR testing, China mandated on October 29th, 2020, that international visitors have both negative RT-PCR and immunoglobulin M (IgM) serology tests, which are part of the "green health code" biological passport, within $48 \mathrm{~h}$ of boarding a direct flight to China [23]. The rationale for this strategy is presumably to broaden the detection window for acute COVID-19 infection by using IgM as an early serology marker, as some people may test negative for COVID-19 RT-PCR several weeks or more after infection but still test positive for COVID-19 IgM serology [23,24].

Restricting travel from highly infected countries at the beginning of a containment phase of a pandemic, allows countries affected to put into place-sustained pandemic containing response measures, so they can implement effective measures to prevent the spread of the pandemic. Sealing of borders and limiting infected passengers are pertinent public health measures in managing a pandemic [1]. Active surveillance, early identification and quarantining of infected individuals with proper contact tracing and preventing further spread of the COVID-19 infection should be the key measures being implemented by the public health authorities in every country affected [1]. Countries should also have protocols and policies in place to identify and quarantine individuals who have repatriated their citizens from other countries, to prevent further spread of COVID-19 from the inbound passengers. Our retrospective analysis showed that out of a total of 2,172 asymptomatic passengers who were screened by the RT-PCR-COVID- 19 test, 57 passengers $(2.62 \%)$ were tested positive for COVID-19. In this study, we found that the RT-PCR test helped to prevent the spread of COVID-19 to 12 other countries amongst the cohort tested (Figure 2). The breakdown of the nationalities of the tested passengers, who were being flown back to their respective countries by repatriation flights out of Doha, shows that the positive passengers were hoping to travel to Sudan, Tunisia, China, Kenya, India, Egypt, Philippines, Morocco, Uganda, Kenya, UK and Syria.

New Zealand last year, recorded their first case of COVID-19, after being COVID free for 102 consecutive days in Auckland on August 11th, 2020 [25]. It hence goes without doubt to show how important it is to screen passengers with proper and stringent screening measures to avoid similar spurt of COVID-19 happening in other countries, which are relatively better controlled in terms of the number of COVID positive patients. It proves beyond doubt that sealing the borders is pertinent to win against COVID-19 pandemic and having a robust screening system in place for passengers is necessary to curb the spread of this virus.

The R0 value is an estimation of the infectivity of the virus measuring how quickly it spreads and the average number of people infected by one infected person. As per world health organisation estimates, R0 value for COVID-19 is between 2-3 [18], which is quite high and hence early diagnosis and detection is pertinent and crucial, to prevent further spread of the virus and halt the pandemic. With an incubation period of up to 14 days, $40-45 \%$ of people infected by the COVID-19 are likely to be asymptomatic but can still silently infect others during this period of infectivity and can have sub-clinical abnormalities on high resolution computed tomography of the chest [26]. Hence, any gaps in the screening process would mean that the spread of COVID-19 will continue to happen with countries being affected one after another [27]. If the number of positive passengers identified in this study were extrapolated considering the R0 values, one infected patient could potentially infect 406 people in a month's time. At this rate, 57 infected passengers from RAK-HC could then possibly infect 23,142 people across twelve countries in 30 days, if not identified and quarantined at the screening stage.

This is obviously a significant number of positive passengers identified and quarantined in this cohort. If this screening process would have been implemented much earlier during the early spread of COVID-19 by all countries, COVID-19 would have perhaps been confined to Wuhan, which was the epicentre of the outbreak and the pandemic would never have unfurled causing so much mortality and morbidity across the globe alongside economic catastrophe, which has crippled the world over. Nowadays, there are thousands of different COVID variants floating around the world. Viruses mutate all the time and the vast majority of the changes are minor. Some of them are even harmful to the virus. Others, on the other hand, can increase the disease's infectiousness or danger - and these mutations tend to predominate. Those with the most concerning changes are referred to as "variants of concern," and they are closely monitored by health officials [3]. They are as follows: i) The India or $\delta$-variant (i.e., B.1.617.2), which has been linked to over 12,000 cases in the UK; ii) The UK, Kent, or $\alpha$-variant (i.e., B.1.1.7) has spread to more than 50 countries and appears to be mutating again; iii) The South Africa or $\beta$-variant (i.e., B.1.351) has been identified in at least 20 countries; and iv) The Brazil or $\gamma$-variant (i.e., P.1) has spread to more than 10 countries [28]. However, it is also unknown whether the vaccines currently being distributed provide protection against the newly identified strains of COVID-19, which is very concerning. Depending on how the variants develop, they could be used to provide a booster vaccine to older or clinically vulnerable people later in the year [28].

With a good vaccination campaign and with a strong screening protocol for passengers oversea, the world will be able to mitigate this pandemic and limit the spread of those new strains. Further studies are recommended to highlight how all countries should adhere to similar screening procedures, identify and quarantine passengers who test COVID positive. This will prevent further spread of COVID-19, help curb the pandemic and most importantly the negative impact on human's lifestyle and get back to normalcy.

Given the synergistic influence of vaccination campaign pooled with passengers' surveillance screening, the world will optimistically be able to limit the spread of the pandemic and the emergence of new strains [28,29]. Further studies are recommended to highlight the adherence of different countries to similar screening protocol, case identification and passengers' quarantine for those tested COVID-19 positive. The effect of such measures is extended beyond the emergency preparedness and management plan of the pandemic to mark a significant step on 
the long road back to normalcy, reversing the negative impact of COVID-19 in humans [30-33].

The present study has one major limitation related to its retrospective design [34]. While retrospective studies save on funds and time, they are marred by their fallacies, since they depend on data that were entered into a clinical database and not collected for research. In our study, certain variables that have the potential to impact the outcome may not have been recorded at all.

\section{CONCLUSION}

Rapid identification of COVID-19 infection is crucial to prevent further spread of this pandemic. The present findings for testing COVID-19 with RT-PCR, helped to improve the effectiveness in containing the outbreak by identifying and preventing travel of infective individuals. This study may contribute to suggest prospective improvements in screening policy for similar future emerging infections and highlight how important it is for countries to have a robust system in place to check for passengers and seal their borders quite early on, to even prevent an epidemic from transforming into a pandemic.

\section{REFERENCES}

1. Varma A, Dergaa I, Ashkanani M, Musa S, Zidan M (2021) Analysis of Qatar's successful public health policy in dealing with the COVID-19 pandemic. International Journal of Medical Reviews and Case Reports 5(2): 6-11

2. Dergaa I, Varma A, Tabben M, Malik RA, Sheik S, et al. (2021) Organising football matches with spectators during the COVID-19 pandemic: What can we learn from the amir cup football final of Qatar 2020? A call for action. Biology of Sport 38(4): 677-681.

3. Gómez CE, Perdiguero B, Esteban M (2021) Emerging SARS-CoV-2 variants and impact in global vaccination programs against SARS-CoV-2/ COVID-19. Vaccines 9(3): 243.

4. (2021) Worldometer COVID-19 coronavirus pandemic.

5. Cellan-Jones R (2020) China launches coronavirus 'close contact detector' app. BBC News.

6. Varma A, Dergaa I, Zidan M, Chaabane M (2020) COVID-19: "Drive thru swabbing hubs" safe and effective testing for travellers. The Journal of Medical Research 6(6): 311-312.

7. Varma A, Abubaker M, Dergaa I (2020) Extensive saliva based COVID-19 testing-the way forward to curtail the global pandemic. The Journal of Medical Research 6(6): 309-310.

8. Mohammed AR (2020) Should all patients having planned procedures or surgeries be tested for COVID-19. American Journal of Surgery and Clinical Case Reports 2(2): 1-3.

9. The Guardian (2020) Coronavirus quarantine precautions around the world.

10. Gostic KM, Kucharski AJ, Lloyd-Smith JO (2015) Effectiveness of traveller screening for emerging pathogens is shaped by epidemiology and natural history of infection. Elife 4: e05564.

11. Bitar D, Goubar A, Desenclos JC (2009) International travels and fever screening during epidemics: a literature review on the effectiveness and potential use of noncontact infrared thermometers. Eurosurveillance 14(6): 19115.

12. da Silva SJR, Pena L (2021) Collapse of the public health system and the emergence of new variants during the second wave of the COVID-19 pandemic in Brazil. One Health 13: 100287.

13. Varma A, Dergaa I, Mohammed AR, Abubaker M, Al Naama A, et al. (2021) COVID-19 and diabetes in primary care- How do hematological parameters present in this cohort? Expert Review of Endocrinology \& Metabolism 16(3): 147-153.
14. Dinnes J, Deeks JJ, Berhane S, Taylor M, Adriano A, et al. (2021) Rapid, point-of-care antigen and molecular-based tests for diagnosis of SARSCoV-2 infection. Cochrane Database of Systematic Reviews 26: 8(8).

15. Bullard J, Dust K, Funk D, Strong JE, Alexander D, et al. (2020) Predicting infectious severe acute respiratory syndrome coronavirus 2 from diagnostic samples. Clinical infectious diseases 71(10): 2663-2666.

16. Humble RM, Merrill AE, Ford BA, Diekema DJ, Krasowski MD (2021) Practical considerations for implementation of SARS-CoV-2 serological testing in the clinical laboratory: experience at an academic medical center. Academic Pathology 8: 23742895211002802.

17. Bohn MK, Lippi G, Horvath A, Sethi S, Koch D, et al. (2020) Molecular, serological and biochemical diagnosis and monitoring of COVID-19: IFCC taskforce evaluation of the latest evidence. Clinical Chemistry and Laboratory Medicine (CCLM) 58(7): 1037-1052.

18. Viceconte G, Petrosillo N (2020) COVID-19 R0: Magic number or conundrum? Infect Dis Rep 12(1): 8516.

19. Musa S, Al Baker W, Al Muraikhi H, Nazareno D, Al Naama A, et al. (2021) Wellness program within primary health care: How to avoid "no show" to planned appointments? A patient-centred care perspective. Physical Activity and Health 5(1): 76-86.

20. Imborek KL, Krasowski MD, Natvig P, Merrill AE, Diekema DJ, et al. (2021) Experience with pretravel testing for SARS-CoV-2 at an academic medical center. Academic Pathology 8: 23742895211010247.

21. Ward S, Lindsley A, Courter J, Assa'ad A (2020) Clinical testing for COVID-19. Journal of Allergy and Clinical Immunology 146(1): 23-34.

22. Kiang MV, Chin ET, Huynh BQ, Chapman LA, Rodríguez-Barraquer I, et al. (2021) Routine asymptomatic testing strategies for airline travel during the COVID-19 pandemic: a simulation study. The Lancet Infectious Diseases.

23. Embassy of the people's Republic of China in the United States of America (2020) Notice on airline boarding requirements for certificates of negative nucleic acid and anti-body blood tests results.

24. Yi X, Chan CW, Yeo KTJ (2021) Is adding IgM antibody to polymerase chain reaction testing useful for COVID-19 travel screening? Am J Clin Pathol 155(3): 321-323.

25. Praveen M (2020) New cases end New Zealand's 'COVID-free' status, Auckland back in lockdown. Healthcare \& Pharma.

26. Oran DP, Topol EJ (2020) Prevalence of asymptomatic SARS-CoV-2 infection: A narrative review. Annals of Internal Medicine 173(5): 362367.

27. World Health Organization (2020) Key considerations for repatriation and quarantine of travellers in relation to the outbreak of novel coronavirus 2019-nCoV.

28. Musa S, Dergaa I, Abdulmalik MA, Ammar A, Chamari K, et al. (2021) BNT162b2 COVID-19 vaccine hesitancy among Parents of 4023 Young Adolescents (12-15 Years) in Qatar. Vaccines 9(9): 981.

29. Dergaa I, Abdelrahman H, Varma A, Yousfi N, Souissi A, et al. (2021) COVID-19 vaccination, herd immunity and the transition toward normalcy: Challenges with the upcoming sports event. Annals of Applied Sport Science 9(3): 10 .

30. Trabelsi K, Ammar A, Masmoudi L, Boukhris O, Chtourou H, et al. (2021) Globally altered sleep patterns and physical activity levels by confinement in 5056 individuals: ECLB COVID-19 international online survey. Biology of Sports 38(4): 495-506.

31. Trabelsi K, Ammar A, Masmoudi L, Boukhris O, Chtourou H, et al. (2021) Sleep quality and physical activity as predictors of mental wellbeing variance in older adults during COVID-19 lockdown: ECLB COVID-19 international online survey. International Journal of Environmental Research and Public Health 18(8): 4329.

32. Musa S, Dergaa I, Mansy O (2021) The puzzle of autism in the time of COVID 19 pandemic: "Light it up Blue". Psychology and Education Journal 58(5): 1861-1873. 
33. Abdulrahman H, Afify EM, Mohammed AS, Malik RA, Dergaa I (2021) Common dermatological complications of COVID 19: How does it affect the skin? Open Access Journal of Biomedical Science 4(2): 1034-1038.

34. Talari K, Goyal M (2020) Retrospective studies-utility and caveats. JR Coll Physicians Edinb 50(4): 398-402., Deeks JJ, Berhane S, Taylor M,
Adriano A, et al. (2021) Rapid, point-of-care antigen and molecularbased tests for diagnosis of SARS-CoV-2 infection. Cochrane Database of Systematic Reviews 26: 8(8). 